\title{
El medio interno del adulto mayor comparado con el del adulto joven de pacientes que acuden al servicio de emergencia de un hospital general de Lima-Perú, estudio de casos y controles
}

\section{Electrolyte and acid-base homeostasis in the elderly compared to that of young patients in an Emergency Service in a General Hospital in Lima, Peru. A case-control study}

Correspondencia Germán Málaga MD, german.malaga@upch.pe

Recibido: 07/06/2017

Arbitrado por pares

Aprobado: 17/05/2017

Citar como: Alva-Rodriguez D, Ciezo J, Málaga G. El medio interno del adulto mayor comparado con el del adulto joven de pacientes que acuden al Servicio de Emergencia de un Hospital General de Lima-Perú. Estudio de casos y controles. Acta Med Peru. 2017;34(2):101-5
Delia Alva-Rodriguez ${ }^{1,3}$, Javier Cieza 2,4 , Germán Málaga $a^{1,3,4}$

1 Servicio de Medicina Interna, Departamento de Medicina, Hospital Cayetano Heredia. Lima, Perú.

2 Servicio de Nefrología, Departamento de Medicina, Hospital Cayetano Heredia. Lima, Perú.

3 Unidad de Conocimiento y Evidencia, Universidad Peruana Cayetano Heredia. Lima, Perú.

4 Facultad de Medicina, Universidad Peruana Cayetano Heredia. Lima, Perú.

\section{RESUMEN}

Objetivo: Estudiar si existe diferencia en alteraciones del medio interno de pacientes adultos mayores en comparación con los adultos jóvenes incidentes a la emergencia de un hospital general en Lima. Materiales y métodos: Se realizó un estudio de casos y controles en 117 pacientes: 57 fueron adultos mayores de 65 años y 62 adultos menores de 65 años, tomados por muestreo accidental y que acudieron entre setiembre y octubre del 2014. En todos los casos y controles, se hizo medición de creatinina y electrolitos séricos al momento de la admisión. Según requerimiento del médico tratante se obtuvo valores de urea, calcio, fosforo, gasometría arterial y proteínas séricas. Se evaluó las variables clínicas y de laboratorio que correlacionaron con las alteraciones de los electrolitos y gases arteriales y se comparó el resultado entre ambos grupos. Resultados: No se halló diferencia entre los valores de electrolitos y gasometría. En las características de base, el grupo de adultos mayores tuvo mayor incidencia de hipertensión arterial (odds ratio [OR]: 2,43; intervalo de confianza al 95\% [IC 95\%]: 1,06-5,57). Únicamente se halló diferencia en los valores de las proteínas séricas totales $(5,40 \pm 0,97$ adultos mayores vs $5,92 \pm 0,95$ adultos jóvenes) $(p=0,030)$. La única variable asociada con cambios en los valores de electrolitos y fósforo sérico, fue tener creatinina sérica $\geq 2 \mathrm{mg} / \mathrm{dl}$ (OR ajustado: 2,67; IC 95\%: 1,21-7,06), independientemente de la edad. Conclusión: La edad no se relacionó con alteraciones del medio interno en los pacientes que acudieron a emergencia. La única variable que mostró efecto en el comportamiento del medio interno fue el tener "creatinina sérica $\geq 2 \mathrm{mg} / \mathrm{dL}^{\text {". }}$

Palabras clave: Adulto mayor; Adulto joven; Electrólitos; Homeostasis (fuente: DeCS BIREME). 


\begin{abstract}
Objective: To determine whether there are differences in the electrolyte and acid-base homeostasis in the elderly compared to that in young adult patients in an emergency service in a general hospital in Lima, Peru. Materials and methods: A case-control study including 117 patients was performed. Fifty-seven were elderly subjects, older than 65 years, and 62 were adults less than 65 years old. Subjects were surveyed using incidental sampling between September and October 2014. In all cases and controls serum creatinine and electrolytes were measured at the time of admission. According to the discretion of the treating physician, serum urea, calcium, phosphorus, arterial blood gases ( $A B G)$, and serum proteins were measured. Clinical and laboratory variables correlating with electrolyte and arterial blood gases alterations were assessed and results were compared between groups. Results: No differences were found between serum electrolyte and arterial blood gases values. With respect to baseline characteristics, the elderly group had a greater frequently of high blood pressure (odds ratio [OR]: 2,43; $95 \%$ confidence interval [IC 95\%]: 1,06-5,57). The only significant difference between the two studied groups was found in the serum total protein values $(5.40 \pm 0.97 \mathrm{~g} / \mathrm{L}$ in the elderly vs. $5.92 \pm 0.95 \mathrm{~g} / \mathrm{L}$ in young patients) $(p=0.030)$. The only variable related to changes in values of serum electrolytes and phosphorus was having serum creatinine $\geq 2 \mathrm{mg} / \mathrm{dL}$, (adjusted OR: $2.67 ; 95 \% \mathrm{Cl}: 1.21-7.06$ ), regardless of age. Conclusion: Age was not associated with changes in the electrolyte or acid-base homeostasis in patients attending the emergency service of a public hospital in Lima. The only variable affecting the behavior of the electrolyte or acid-base homeostasis, was having a serum creatinine $\geq 2 \mathrm{mg} / \mathrm{dl}$.
\end{abstract}

Keywords: Aged; Young adult; Electrolytes; Homeostasis (source: MeSH NLM).

\section{INTRODUCCIÓN}

Los pacientes adultos mayores tienen una mayor frecuencia de alteraciones del medio interno, es una verdad que se asume sin discusión ${ }^{[1,2]}$ y las causas determinantes serían la edad y los cambios estructurales y funcionales renales propias del envejecimiento ${ }^{[3]}$, menor control de los volúmenes intra y extracelular ${ }^{[4]}$, disminución de los mecanismos para el balance electrolítico, disminución en la tasa de filtrado glomerular o mayor dificultad en el manejo del sodio y en la capacidad de concentración y acidificación de la orina ${ }^{[5,6]}$. Sin embargo, las alteraciones electrolíticas pueden estar estrechamente relacionadas a otros factores como la presencia de comorbilidades, especialmente afectaciones renales y uso frecuente de fármacos como los diuréticos. Situaciones usuales en edades avanzadas que con frecuencia no se encuentran igualmente ponderadas en adultos menores.

Los desbalances electrolíticos son situaciones frecuentes que se asocian a mayor mortalidad en los pacientes hospitalizados en todas las edades ${ }^{[7-12]}$. Localmente pueden estar presentes hasta en el $60 \%$ de los pacientes hospitalizados ${ }^{[13]}$ y en la mayoría de estudios la hiponatremia es descrita, como la alteración más frecuente ${ }^{[14-16]}$. A nivel local, se ha encontrado además una frecuencia alta para las alteraciones del potasio $(38,7 \%)$, siendo casi tan frecuente como las alteraciones del sodio $(40,1 \%)$ [13]. Esto hace de los desbalances electrolíticos un problema importante, cuya identificación y manejo oportuno son determinantes para la recuperación de los pacientes, que han sido descritos de forma limitada a nivel local y que posiblemente presenta características diferentes a lo presentado en estudios de otros entornos.

A nivel local, existe un aumento progresivo y sostenido de la demanda de pacientes adultos mayores en los servicios de emergencia ${ }^{[17]}$, como consecuencia del incremento poblacional, la transición demográfica y un sistema de salud del primer nivel deficiente que no aporta en la necesaria contención a la creciente demanda. Este problema frecuente al que se enfrentan los profesionales de salud local, aún no ha sido suficientemente abordado ni descrito.

El objetivo de este estudio fue comparar la situación del medio interno del paciente adulto mayor y la del adulto joven que acuden a la emergencia y requieren manejo intrahospitalario en un hospital que atiende una alta y compleja demanda de pacientes en Lima Metropolitana.

\section{MATERIALES Y MÉTODOS}

Se realizó un estudio observacional comparativo de un grupo de casos constituido por pacientes adultos mayores y otro de controles constituido por pacientes adultos jóvenes, procedentes del cono norte de la ciudad, que acuden a la emergencia del Hospital Nacional Cayetano Heredia entre setiembre y octubre del año 2014 por muestreo accidental. Usamos la "variable predictora", es decir el supuesto factor de riesgo como elemento de discernimiento para la selección de casos y controles.

Fueron incluidos todos los pacientes quienes cumplían con los siguientes criterios: mayores de 18 años, requerimiento de asistencia médica a la emergencia y que a juicio del médico requiriera ser observado por más de 24 horas en el hospital y a quienes se les hubiera solicitado urea, creatinina y electrolitos séricos al momento del ingreso a la sala de observación en la emergencia, que por norma general se les solicita a todas las personas que van a ser hospitalizadas. No se excluyo a ningún paciente por su condición previa de enfermedad de cualquier índole. Se excluyeron pacientes anúricos, que hubieran sido tratados directamente en la unidad de shock trauma por su condición de gravedad extrema, gestantes, pacientes con requerimientos quirúrgicos o traumatológicos de emergencia o urgencia y aquellos que hubieran sido derivados de otro centro de atención de salud (hospital, centro de salud o equivalentes) y que hubieran sido sujetos de manejo médico previo. 
Tabla 1. Características demográficas y comorbilidades por grupos de comparación.

\begin{tabular}{lccc} 
Características & $\begin{array}{c}\text { Adultos } \\
\text { mayores } \\
\mathbf{N = 5 7} \\
\mathbf{n} \%\end{array}$ & $\begin{array}{c}\text { Adultos } \\
\text { jóvenes } \\
\mathbf{N = 6 2} \\
\mathbf{n} \%\end{array}$ & $\begin{array}{c}\text { Valor } \\
\boldsymbol{p}^{*}\end{array}$ \\
$\begin{array}{lccc}\text { Sexo } \\
\text { Hombre }\end{array}$ & $29(50,88)$ & $24(38,71)$ & \\
$\quad$ Mujer & $28(49,12)$ & $38(61,29)$ & \\
Comorbilidades & & & \\
$\quad$ Diabetes mellitus & $6(10,53)$ & $11(17,74)$ & 0,261 \\
Hipertensión arterial & $21(36,84)$ & $12(19,35)$ & 0,033 \\
$\quad$ Uropatía obstructiva & $15(26,32)$ & $11(17,74)$ & 0,258 \\
Antecedentes & & & \\
$\quad$ Enfermedad renal & $11(19,30)$ & $11(17,74)$ & 0,827 \\
Cáncer & $5(8,77)$ & $4(6,45)$ & 0,447 \\
Uso de sonda Foley & $14(24,56)$ & $9(14,52)$ & 0,331 \\
\hline
\end{tabular}

* Análisis estadístico: cálculo de OR para cada una de las variables dependientes e independientes

Se definieron a priori dos grupos tomando en cuenta el factor de riesgo que significa la edad, así se definieron dos grupos: uno de pacientes de 65 años o mas (casos) y otro de pacientes menores de 65 años (controles). Por cada paciente enrolado como caso ingresamos al paciente inmediato posterior al grupo control. Se calculó un tamaño de muestra con un intervalo de confianza de 95\% (IC 95\%), una potencia del 80\%, una desviación estándar de la muestra de 0,2 y una diferencia esperable entre valores de $0,1 \mathrm{mg} / \mathrm{dL}$ de creatinina sérica. El tamaño de muestra obtenido, calculado con el programa "Epi-info".

El estudio fue aprobado por el Comité de Ética institucional del Hospital donde fue realizado el estudio. Para incluir al paciente se solicitó un consentimiento informado que incluyó la autorización para acceder a su historia clínica de emergencia para obtener la información necesaria, como datos de filiación, comorbilidades relevantes para el estudio e información requerida para el estudio. Los datos de filiación obtenidos fueron: edad, sexo, y tiempo de hospitalización. Las comorbilidades y antecedentes evaluados fueron hipertensión arterial, diabetes mellitus, cáncer, enfermedad renal, uropatía obstructiva y uso de dispositivos intravesicales (uso de Sonda Foley). De los resultados de laboratorio, se registraron los valores séricos de creatinina, urea, sodio, potasio, cloro; calcio, fósforo, albúmina, proteínas totales y valores de gases arteriales, así como densidad urinaria, $\mathrm{pH}$ urinario y el resultado del urocultivo cuando fue solicitado.

El análisis estadístico fue realizado utilizando el programa estadístico SPSS V.13. El análisis bivariado entre las características demográficas y comorbilidades versus el grupo de estudio fue utilizando la Prueba Chi cuadrado y la Prueba Exacta de Fisher. Para el análisis de las variables continuas por grupo de estudio usamos la Prueba t de Student para muestras independientes. Se hizo cálculo de Odds-Ratio (OR) para establecer el peso de las variables, comorbilidades y establecer asociaciones. Para definir las variables que influencian los trastornos del medio interno (sodio, potasio, cloro, fósforo, calcio y gases sanguíneos) se realizó análisis de regresión multivariado para cada electrolito en función de la edad, el tipo de comorbilidad como diabetes, hipertensión arterial, cáncer o uropatía obstructiva y de la función renal. Para considerar existencia de diferencia se consideró como estadísticamente significativo un valor de $p<0,05$.

\section{RESULTADOS}

Fueron seleccionados 63 pacientes por grupo. De los 126 pacientes incluidos en el estudio, seis fueron retirados del grupo de adultos mayores y uno del grupo de adultos jóvenes por no presentar el resultado de creatinina o electrolitos.

Los datos de filiación y comorbilidades son presentados en la Tabla 1. La edad promedio del grupo de adultos mayores fue de $73,4 \pm 11,8$ años y la edad promedio del grupo de adultos jóvenes fue de $44,9 \pm 16,1$ años. El grupo de adultos mayores tuvo mayor proporción de pacientes con hipertensión arterial que el grupo de adultos jóvenes, con un OR de 2,43 (IC 95\%: $1,06-5,57)$. No hallamos diferencias significativas en el resto de variables evaluadas.

La Tabla 2 compara las variables asociadas al medio interno entre los grupos de estudio. Únicamente las proteínas totales mostraron diferencia estadísticamente significativa, siendo el mayor valor para los adultos jóvenes y el menor para los adultos mayores. Ninguno de los valores de electrolitos, gases, creatinina o urea mostró diferencias estadísticamente significativas. Para definir las variables que influencian los trastornos del medio interno (sodio, potasio, cloro, fósforo, calcio y gases sanguíneos) se realizó análisis de regresión multivariado para cada electrolito en función de la edad, el tipo de comorbilidad como diabetes, hipertensión arterial, cáncer o uropatía obstructiva y de la función renal.

La disfunción renal, definida con un valor creatinina mayor de 2 $\mathrm{mg} / \mathrm{dL}$ (OR ajustado: 2,67; IC 95\%: 1,21-7,06), se relacionó con valores significativamente diferentes de sodio, potasio, cloro y fósforo. En cambio, ni la edad o el tipo de comorbilidad clínica, mostraron esta relación, lo que puede ser apreciado en la Tabla 3.

\section{DISCUSIÓN}

Nuestro estudio sugiere que el medio interno del adulto mayor, a pesar de los cambios fisiológicos descritos que supondrían per se, un factor de riesgo ${ }^{[2,3]}$ no difiere del medio interno del adulto joven al ser admitido por una enfermedad aguda en la emergencia. Esto rechazaría la hipótesis de que los adultos mayores por tener una disminución del agua corporal total, tener una mayor prevalencia poblacional de hiponatremia ${ }^{[2]}$ tener los mecanismos de protección como el reflejo de la sed alterados ${ }^{[3]}$ y muy probablemente una función renal disminuida, fenómenos propios del envejecimiento, ${ }^{[18]}$ serían más proclives al desarrollo de alteraciones de su medio interno ante injurias agudas equivalentes. 
Tabla 2. Medio interno por grupos de estudio.

\begin{tabular}{lccc}
\multicolumn{1}{c}{ Características } & $\begin{array}{c}\text { Adultos } \\
\text { mayores } \\
\text { N=57 } \\
\text { Media } \pm \text { DE* }\end{array}$ & $\begin{array}{c}\text { Adultos } \\
\text { jóvenes } \\
\text { N=62 } \\
\text { Media } \pm \text { DE* }\end{array}$ & $\begin{array}{c}\text { Valor } \\
\boldsymbol{p}^{\dagger}\end{array}$ \\
\hline Urea (mg/dL) & $43,37 \pm 38,18$ & $41,68 \pm 37,81$ & 0,810 \\
Creatinina (mg/dL) & $0,86 \pm 0,77$ & $0,98 \pm 0,89$ & 0,435 \\
Sodio (mEq/L) & $134,65 \pm 6,88$ & $136,03 \pm 5,21$ & 0,218 \\
Potasio (mEq/L) & $4,05 \pm 0,79$ & $4,09 \pm 0,67$ & 0,766 \\
Cloro (mEq/L) & $102,77 \pm 9,05$ & $103,45 \pm 6,82$ & 0,643 \\
pH arterial & $7,39 \pm 0,08$ & $7,40 \pm 0,12$ & 0,638 \\
HCO3- arterial (mEq/L) & $20,40 \pm 5,70$ & $20,59 \pm 4,49$ & 0,860 \\
pCO2 arterial (mmHg) & $33,34 \pm 9,00$ & $31,34 \pm 6,74$ & 0,232 \\
pO2 (mmHg) & $91,48 \pm 30,20$ & $96,76 \pm 34,22$ & 0,434 \\
Calcio total (mg/dL) & $7,72 \pm 1,04$ & $7,91 \pm 0,73$ & 0,452 \\
Fósforo (mg/dL) & $3,47 \pm 0,80$ & $3,36 \pm 1,69$ & 0,799 \\
Albúmina(g/dL) & $2,46 \pm 0,63$ & $2,79 \pm 0,86$ & 0,064 \\
Proteínas totales (g/dL) & $5,40 \pm 0,97$ & $5,92 \pm 0,95$ & 0,030
\end{tabular}

* DE: Desviación estándar; $\mathrm{HCO}$ : Bicarbonato de sodio; pCO2: presión de dióxido de carbono; pO2: presión de oxígeno.

† Análisis estadístico: t-Student para dos muestras con igual varianza

Como consecuencia de la técnica del muestreo accidental (muestreo no probabilístico), no se logró controlar todas las variables, lo que generó diferencias en las condiciones basales entre ambos grupos, por ejemplo, el grupo del adulto mayor estuvo cargado con pacientes hipertensos lo que pudo devenir en una variable de confusión, esta diferencia estaría traduciendo la mayor prevalencia de hipertensión arterial en el adulto mayor, llegando en algunas series a bordear el $80 \%{ }^{[19]}$, mientras que en la población general la prevalencia de hipertensión arterial en Perú, estaría alrededor del $24 \%{ }^{[20]}$, entonces, es posible que el muestreo accidental este reflejando dicha diferencia. En todo caso, esta situación que pudo haber favorecido una mayor frecuencia $y / o$ gravedad de las alteraciones del medio interno ${ }^{[21,22]}$, no tendría sustento de acuerdo con los resultados de nuestro estudio.

El factor más importante hallado como causante de la perturbación del medio interno, fue la presencia de afección de la función renal, cuya significancia debe corresponder a una Tasa de Filtrado Glomerular (TFG) $<45 \mathrm{cc} / \mathrm{min}$ para cualquier edad, lo que corresponde aproximadamente a valores de creatinina $\geq 2 \mathrm{mg} / \mathrm{dl}^{[5]}$, la asociación correspondió a un OR de 2,67 veces. En tal situación, tanto adultos mayores como jóvenes muestran variaciones estadísticamente significativas en la concentración del sodio, potasio, cloro y fósforo, mas no en los valores de la gasometría arterial. Estos resultados permiten inferir que, ante una afección renal, las alteraciones del medio interno serán más frecuentes y graves de manera independiente a la edad (alteraciones secundarias o consecuentes que encontramos en FRA/ERC). Entonces, es posible que al ser la enfermedad renal crónica mucho más prevalente en el adulto mayor ${ }^{[2]}$ se habría generado incorrectamente la inferencia de que las alteraciones del medio interno son más frecuentes en la población adulta mayor, dadas sus desventajas fisiológicas. Sin embargo, esta situación no ha sido avalada por los resultados obtenidos en el presente estudio.

Como parte del proceso normal de envejecimiento, es de esperar que los adultos mayores tuvieran menores niveles de proteínas totales y albúmina sérica ${ }^{[23]}$, situación ratificada en el presente estudio, sin embargo, esta aparente desventaja ${ }^{[24]}$ no se tradujo o relacionó con mayores alteraciones del medio interno. Sin embargo, es posible que en situaciones extremas el resultado pudiera ser distinto, pues considerando que la fisiología renal es dependiente del flujo sanguíneo y volumen circulante efectivo el que es dependiente de los niveles de proteínas ${ }^{[25]}$ y que cualquier injuria que afecte la circulación sistémica y renal generará efectos no deseables en la fisiología del riñón haciéndolo susceptible sufrir de injuria inflamatoria aguda, lo que para un adulto mayor con desnutrición crónica pudiera ser una desventaja ${ }^{[23,24,26]}$.

Queremos puntualizar que en pacientes adultos mayores que acuden a la emergencia (deben tenerse en cuenta las características específicas de este grupo etario y valorarse diferentes esferas para evitar o disminuir/atenuar complicaciones habituales en este grupo a través de una valoración geriátrica breve), es más importante considerar el tipo y magnitud de la injuria por un lado y las características del huésped, dentro de las que debe resaltarse por sobre todo la función renal y probablemente el estado nutricional para efectos de alteraciones del medio interno, más que la edad per se. En relación a este último concepto el mensaje es claro, todo paciente incidente a la emergencia de un hospital local debería tener obligatoriamente un control de la creatinina sérica que permita establecer si el paciente tiene disfunción (deterioro de la función) renal, la que se asocia con aumento del riesgo complicaciones y de muerte ${ }^{[24]}$, lo que sería independiente de la edad del paciente.

Nuestro estudio tiene algunas limitaciones, como son: el muestreo accidental no probabilístico que podría generar sesgos de selección, el tamaño de la muestra que no permitió controlar todas las variables, el hecho de que haya sido realizado en una sola institución lo que hace que sus resultados sean no generalizables, al tratarse de una recolección en un periodo de tiempo, las inferencias estacionales pudieran introducir algún tipo de sesgo de selección. Otra potencial limitación está relacionada con el tipo de estudio, pues, al no haber seguido a estos pacientes, es posible que las variables exploradas como factores de riesgo, tengan evoluciones distintas en el curso de la hospitalización. Finalmente, no hicimos evaluación nutricional en los pacientes, por lo que no podemos explicar la diferencia en los niveles de proteínas hallada entre ambos grupos. Entre las fortalezas mencionaremos, la naturaleza pionera de nuestra observación, pues no hemos hallado estudios similares en la literatura internacional consultada.

Concluimos que no existen diferencias del medio interno en relación con la edad de los pacientes adultos jóvenes y adultos 
Tabla 3. Electrolitos y gasometría arterial en función de la creatinina sérica.

\begin{tabular}{lccc} 
Características & $\begin{array}{c}\text { Creatinina } \\
<2 \mathrm{mg} / \mathbf{d L} \\
\mathbf{N}=108 \\
\text { Media } \pm \text { DE* }\end{array}$ & $\begin{array}{c}\text { Creatinina } \\
\mathbf{2} \mathbf{m g} / \mathbf{d L} \\
\mathbf{N = 1 1} \\
\text { Media } \pm \text { DE* }\end{array}$ & $\begin{array}{c}\text { Valor } \\
\boldsymbol{p}^{+}\end{array}$ \\
\hline Edad (años) & $59,14 \pm 19,81$ & $53,09 \pm 22,98$ & 0,344 \\
Sodio (mEq/L) & $134,83 \pm 5,96$ & $140,64 \pm 4,82$ & 0,002 \\
Potasio (mEq/L) & $4,00 \pm 0,64$ & $4,78 \pm 1,14$ & 0,001 \\
Cloro (mEq/L) & $102,52 \pm 7,83$ & $109,09 \pm 6,66$ & 0,008 \\
pH arterial & $7,39 \pm 0,10$ & $7,37 \pm 0,10$ & 0,526 \\
HCO3- arterial & $20,81 \pm 4,99$ & $18,12 \pm 5,69$ & 0,101 \\
(mEq/L) & & & \\
pCO2 arterial (mmHg) & $32,65 \pm 8,08$ & $30,25 \pm 7,40$ & 0,352 \\
Calcio total (mg/dL) & $7,79 \pm 0,89$ & $8,13 \pm 0,77$ & 0,369 \\
Fósforo (mg/dL) & $3,18 \pm 1,02$ & $4,70 \pm 1,95$ & 0,007 \\
\hline
\end{tabular}

* DE: Desviación Estándar. HCO3: Bicarbonato de sodio; pCO2: presión de dióxido de carbono; pO2: presión de oxígeno.

† Análisis estadístico: t-Student para dos muestras con igual varianza

mayores (en un hospital nivel III a través de un muestreo accidental o casual). Sin embargo, el tener alteración de la función renal evidenciada con un nivel de creatinina $\geq 2 \mathrm{mg} / \mathrm{dl}$, se asocia con un riesgo de 2,67 veces de tener alguna alteración en el medio interno.

Fuente de financiamiento: autofinanciado.

Conflictos de interés: Los autores declara no tener conflictos de interés.

\section{REFERENCIAS BIBLIOGRÁFICAS}

1. Mannesse CK, Vondeling AM, van Marum RJ, van Solinge WW, Egberts TC, Jansen PA. Prevalence of hyponatremia on geriatric wards compared to other settings over four decades: a systematic review. Ageing Res Rev. 2013;12(1):165-73.

2. Cumming K, Hoyle GE, Hutchison JD, Soiza RL. Prevalence, incidence and etiology of hyponatremia in elderly patients with fragility fractures. PLoS One. 2014;9(2):e88272.

3. Sobamowo $\mathrm{H}$, Prabhakar SS. The kidney in aging: physiological changes and pathological implications. Prog Mol Biol Transl Sci. 2017;146:303-40.

4. Hooper L, Bunn D, Jimoh FO, Fairweather-Tait SJ. Water-loss dehydration and aging. Mech Ageing Dev. 2014;136-137:50-8.

5. Gregori JA, Núñez JF. Handling of water and electrolytes in the healthy old. Rev Clin Gerontol. 2009;19(01):1-2.

6. Luckey AE, Parsa CJ. Fluid and electrolytes in the aged. Arch Surg. 2003;138(10):1055-60.

7. Asadollahi K, Hastings IM, Beeching NJ, Gill GV. Laboratory risk factors for hospital mortality in acutely admitted patients. QJM. 2007;100(8):501-7.
8. Lindner G, Funk GC, Schwarz C, Kneidinger N, Kaider A, Schneeweiss $B$, et al. Hypernatremia in the critically ill is an independent risk factor for mortality. Am J Kidney Dis. 2007;50(6):952-7.

9. McCallum L, Jeemon P, Hastie CE, Patel RK, Williamson C, Redzuan $\mathrm{AM}$, et al. Serum chloride is an independent predictor of mortality in hypertensive patient's novelty and significance. Hypertension. 2013;62(5):836-43.

10. Miura S, Yoshihisa A, Takiguchi M, Shimizu T, Nakamura Y, Yamauchi $\mathrm{H}$, et al. Association of hypocalcemia with mortality in hospitalized patients with heart failure and chronic kidney disease. J Card Fail. 2015;21(8):621-7.

11. Rubeiz GJ, Thill-baharozian M, Hardie D, Carlson RW. Association of hypomagnesemia and mortality in acutely ill medical patients. Crit Care Med. 1993;21(2):203-9.

12. Lewis JR, Hassan SK, Wenn RT, Moran CG. Mortality and serum urea and electrolytes on admission for hip fracture patients. Injury. 2006;37(8):698-704.

13. Meza Huamán A, Cieza Zevallos JA. Frecuencia y características de las alteraciones electrolíticas en pacientes hospitalizados en servicios de Medicina en un hospital general. Rev Med Hered. 2016;27(4):237-42.

14. Balcı AK, Koksal O, Kose A, Armagan E, Ozdemir F, Inal T, et al. General characteristics of patients with electrolyte imbalance admitted to emergency department. World J Emerg Med. 2012;4(2):113-6.

15. Lee CT, Guo HR, Chen JB. Hyponatremia in the emergency department. Am J Emerg Med. 2000;18(3):264-8.

16. Upadhyay A, Jaber BL, Madias NE. Incidence and prevalence of hyponatremia. Am J Med. 2006;119(7):S30-5.

17. Rodríguez T, Varela L, Ortiz J, Chávez H. Estancia hospitalaria y mortalidad en adultos mayores hospitalizados en un hospital general de Lima Metropolitana, 1997-2008. Rev Med Hered. 2011;22 (1):23-8.

18. Jha V, Garcia-Garcia G, Iseki K, Li Z, Naicker S, Plattner B, et al. Chronic kidney disease: global dimension and perspectives. Lancet. 2013;382(9888):260-72.

19. Zattar LC, Boing AF, Giehl MW, d'Orsi E. Prevalence and factors associated with high blood pressure, awareness, and treatment among elderly in Southern Brazil. Cad Saude Publica. 2013;29(3):507-21.

20. Régulo Agusti C. Epidemiología de la hipertensión arterial en el Perú. Acta Med Peru. 2006;23(2):69-75.

21. National High Blood Pressure Education Program. 1995 Update of the working group reports on chronic renal failure and renovascular hypertension. Bethesda, MD: National Institutes of Health, National Heart, Lung, and Blood Institute; 1995.

22. Vaes B, Beke E, Truyers C, Elli S, Buntinx F, Verbakel JY, et al. The correlation between blood pressure and kidney function decline in older people: a registry-based cohort study. BMJ Open. 2015;5(6):e007571.

23. Go AS, Chertow GM, Fan D, McCulloch CE, Hsu CY. Chronic kidney disease and the risks of death, cardiovascular events, and hospitalization. N Engl J Med. 2004;351(13):1296-305.

24. Soderstrom L, Rosenblad A, Adolfsson ET, Saletti A, Bergkvist L. Nutritional status predicts preterm death in older people: a prospective cohort study. Clin Nutr. 2014;33(2):354-9.

25. Kelly IE, Tessier S, Cahill A, Morris SE, Crumley A, McLaughlin D, et al. Still hungry in hospital: identifying malnutrition in acute hospital admissions. QJM. 2000;93(2):93-8.

26. Varela L. Principios de Geriatría y Gerontología. 2da ed. Lima: Centro Editorial UPCH; 2011. 